\title{
Orbiter Aerodynamic Acceleration Flight Measurements in the Rarefied-Flow Transition Regime
}

\author{
Robert C. Blanchard* \\ NASA Langley Research Center \\ Hampton, Virginia 23681-0001 \\ Richard G. Wilmoth $†$ \\ NASA Langley Research Center \\ Hampton, Virginia 23681-0001 \\ Gerald J. LeBeau $\ddagger$ \\ NASA Johnson Space Center \\ Houston, Texas 77058-3696
}

\begin{abstract}
Acceleration data taken from the Orbital Acceleration Research Experiment (OARE) during reentry on STS-62 have been analyzed using calibration factors taken on orbit. This is the first Orbiter mission which collected OARE data during the Orbiter reentry phase. The data examined include the flight regime from orbital altitudes down to about $90 \mathrm{~km}$ which covers the free-molecule-flow regime and the upper altitude fringes of the rarefied-flow transition into the hypersonic continuum. Ancillary flight data on Orbiter position, orientation, velocity, and rotation rates have been used in models to transform the measured accelerations to the Orbiter center-of-gravity, from which aerodynamic accelerations along the Orbiter body axes have been calculated. Residual offsets introduced in the measurements by unmodeled Orbiter forces are identified and discussed. Direct comparisons are made between the OARE flight data and an independent micro-gravity accelerometer experiment, the High Resolution Accelerometer Package (HiRAP), which also obtained flight data on reentry during the mission down to about $95 \mathrm{~km}$. The resulting OARE aerodynamic acceleration measurements along the Orbiter's body axis, and the normal to axial acceleration ratio in the free-molecule-flow and transition-flow regimes are presented and compared with numerical simulations from three direct simulation Monte Carlo codes.
\end{abstract}

*Senior Research Engineer, Aerothermodynamics Branch, Gas Dynamics Division, Associate Fellow AIAA.

†Senior Research Engineer, Aerothermodynamics Branch, Gas Dynamics Division, Senior Member AlAA.

$\ddagger$ Research Engineer, Aeroscience Branch, Aeroscience and Flight Mechanics Division.

Copyright, 1994 by the American Institute of Aeronautics and Astronautics, Inc. No copyright is asserted in the United States under Title 17, U.S. Code. The U.S. Government has a royalty-free license to exercise all rights under the copyright claimed herein for Governmental purposes. All other rights are reserved by the copyright owner.

\section{Nomenclature}

A

$\mathrm{C}_{\mathrm{A}}$

$\mathrm{C}_{\mathrm{N}}$

CY

$\mathrm{g}$

$\mathrm{L}_{\mathrm{ref}}$

$\mathrm{Kn}$

m

$\mathrm{p}, \mathrm{q}, \mathrm{r}$

$S$

$\mathrm{V}_{\mathrm{a}}$

$\mathrm{X}, \mathrm{Y}, \mathrm{Z}$

$\alpha$

$\beta$

$\lambda$

$\rho$

ng

$\mu g$

subscripts

b

$x, y, z$

Acronyms

APU

DSMC

FES

GSE

HiRAP

IMU

ISC

MSID

MET

OARE

OMS

STS acceleration component

axial coefficient

normal coefficient

side force coefficient

gravitational acceleration $\left(9.80665 \mathrm{~m} / \mathrm{s}^{2}\right)$

vehicle reference length, $\mathrm{m}$

Knudsen Number, 1/Lref

vehicle mass, $\mathrm{kg}$

body rotation rates, $\mathrm{sec}^{-1}$

vehicle reference area, $\mathrm{m}^{2}$

air relative velocity, $\mathrm{m} / \mathrm{s}$

sensor or body axes

angle of attack, deg.

side-slip angle, deg.

mean-free-path, $m$

mass density, $\mathrm{kg} / \mathrm{m}^{3}$

nano-g, $1 \times 10^{-9} \mathrm{~g}$

micro-g, $1 \times 10^{-6} \mathrm{~g}$

body axes

coordinate axes

Auxiliary Power Unit

Direct Simulation Monte Carlo

Flash Evaporator System

Ground Support Equipment

High Resolution Accelerometer Package

Inertial Measurement Unit

Johnson Space Center

Measurement / Stimuli Identification

Mission Elapsed Time

Orbital Acceleration Research Experiment

Orbiter Maneuvering System

Shuttle Transportation System 


\section{Introduction}

The Orbital Acceleration Research Experiment ${ }^{1}$ (OARE) is a flight experiment flown on the Shuttle Orbiter which contains a tri-axial accelerometer that uses a single free-floating (non-pendulous) electrostatically suspended cylindrical proof-mass. The accelerometer sensor assembly is mounted to a microprocessor-controlled, dual-gimbal platform in order to perform in-flight calibrations. This feature of the equipment provides for unique insitu calibration factors which significantly improve the precision and confidence of the flight results over using calibration factors performed in a $1-\mathrm{g}$ environment. Several earlier Orbiter missions for which OARE data was collected, analyzed, and reported 2,3 were for the orbital portion of the mission, this is the first OARE data corresponding to the Orbiter's reentry phase.

OARE 's principle objective is to measure Orbiter aerodynamic performance on orbit and during the initial stages of reentry. Thus, the OARE is a nano-g class instrument purposely designed for low-frequency acceleration signals. As the low-frequency acceieration environment contains a variety of components (e.g. gravity-gradient, rotational effects), models of these components embedded in the acceleration measurements are required in order to extract the Orbiter aerodynamic acceleration signal from the flight data. These models require additional flight measurements (e.g. vehicle position, velocity, orientation and angular rates) which are obtained routinely by the Shuttle Project Office as standard ancillary data on each Orbiter mission. The models and measurements required to separate the components of low-frequency acceleration are discussed in the appendix of reference 4. More details on the method used to extract the aerodynamic signals are given in reference 5, although, the main ideas will be outlined in this report to provide continuity.

This report presents the results of the extracted aerodynamic signals from the OARE measurements taken during reentry on the STS- 62 mission. A comparison is presented with data taken from the HiRAP experiment during this flight. The HiRAP is a micro-g class experiment package, the forerunner of the OARE concept, and has flown extesively on the Orbiter since $1983^{6}$. Also, recent computer simulations using three different direct simulation Monte Carlo codes, are compared with the OARE flight data .

\section{Instrument Overview}

A brief overview of the instrument system is given for completeness. A schematic of OARE showing the various instrument components is shown in Fig. 1. The instrument system weighs $53.2 \mathrm{~kg}(117 \mathrm{lbs}$.) and requires 110 watts of power. The OARE system consists of three replaceable units which are mounted to a keel bridge mounting plate. The three units are: (1) the calibration table and sensor package, (2) the interface electronics, power system and servo control modules, and (3) the 16-bit programmable micro-computer and memory. The overall system dimensions are $43.2 \times 33 \times$ $104.1 \mathrm{~cm}(17 \times 13 \times 41 \mathrm{in})$. The OARE is mounted on a keel bridge of the Orbiter at bay 11 on the cargo bay floor, as shown schematically in Fig. 2. The OARE sensor axes are co-aligned with the Orbiter body axes as shown.

The accelerometer sensor (labeled "sensor package" in Fig. 1) is attached to a moveable platform. The platform is moveable about two axes, the inner-gimbal and the outergimbal axis, by two brushless DC torque motors, as illustrated in Fig. 1. There are 3 sensor ranges, A, B, and C, which correspond to acceleration scales of $\pm 10,000, \pm 1000$ and $\pm 100 \mu \mathrm{g}$ respectively for the $X$-axis and $\pm 25,000, \pm 1970$ and $\pm 150 \mu \mathrm{g}$ respectively for the $\mathrm{Y}$ - and $\mathrm{Z}$-axes. The best sensor resolution is $3.05 \mathrm{ng}$ which is along the $\mathrm{X}$-axis. The $\mathrm{Y}$ - and $\mathrm{Z}$ - axes have a slightly larger sensor resolution value of $4.6 \mathrm{ng}$.

\section{Trajectory Information}

For each Orbiter mission, the Orbiter Project Office at JSC provides trajectory data prior to the flight and these data are updated after the flight using measurements taken during the mission. Fig. 3 shows the profiles of the Shuttle Orbiter position, velocity, and orientation. Specifically, Fig. 3(a) shows the altitude and air relative velocity, $\mathrm{V}_{\mathrm{a}}$, for the STS62 mission as a function of MET for the initial portion of the reentry. The altitude is calculated above a reference ellipsoid ${ }^{7}$ from Orbiter Cartesian position data. The values used to calculate the Earth's radius for a given latitude are:

$$
\text { equatorial radius }=6378.145 \mathrm{~km}
$$$$
\text { eccentricity }=0.08182
$$

The data shown cover a time period which corresponds to about 1 hour prior to deorbit burn when the OARE reentry data flag was initiated from the ground flight controllers to about $90 \mathrm{~km}$ in altitude after which the OARE sensors become saturated. The deorbit burn, at about $334.4 \mathrm{~h}$ MET, is clearly evident in Fig. 3. After the deorbit maneuver the Orbiter is placed on a path with a lower periapsis which accounts for the subsequent increase in velocity as the Orbiter approaches its new periapsis. Further down the velocity curve, the more dense atmosphere is encountered which provides another dramatic change in velocity as the Orbiter departs from its conic behavior due to increasing atmospheric effects.

The Orbiter attitude change is shown in Fig. 3(b) in terms of the wind angles, i.e. angle-of-attack, $\alpha$ and side-slip angle, $\beta$. These angles have been calculated from the body quaternion and velocity data sets used by the Orbiter navigation system provided by JSC. Clearly seen on the figure are large changes in $\alpha$ prior to and after deorbit burn. Prior to deorbit burn, the spacecraft maneuvers using its primary and vernier thrusters to place the tail of the vehicle into the direction of motion in order to effect a change in orbit by the OMS rocket motors. After the deorbit burn, the Orbiter performs a large positive pitch maneuver to orient the spacecraft into its preferred entry attitude of $40 \mathrm{deg}$. These motions induce acceleration inputs into the OARE which must be accounted for in order to extract the aerodynamic signal. Also, the rapid change in environment (due to orientation changes) provides a challenge to acquire reliable calibration data. 


\section{Aerodynamic Information}

Based upon the preceding wind angle information for the STS-62 mission, Fig. 4 shows the predicted three body axes free-molecule-flow aerodynamic coefficients, $\mathrm{C}_{\mathrm{A}}, \mathrm{CY}_{\mathrm{Y}}$, $\mathrm{C}_{\mathrm{N}}$ for the entire reentry period under investigation. These data are from the Orbiter Project Office data base ${ }^{7}$ for the condition when Orbiter payload doors are closed. The aerodynamic data are free-molecule-flow calculations generated by dividing the Orbiter surface geometry into multiple flat-plates and assuming fully diffuse surface reflection conditions. There are several points to be made from these graphs. First, the aerodynamic coefficients are not applicable for all the time shown. Particularly, where the coefficients are nearly constant at times greater than about 334.65 $\mathrm{h} \mathrm{MET}$ in Fig.4. This is the region when the vehicle begins to transition from the free-molecule flow to the hypersonic continuum and adjustments must be made to the coefficients shown. Second, there are instances during flight when a given Orbiter body axis is perpendicular to the flow thus providing zero values for its coefficient and, thus, an aerodynamic acceleration adjustment opportunity. This situation provides extremely valuable accelerometer offset checks in the aerodynamic extraction analysis. An example of this is the axial coefficient, $\mathrm{C}_{\mathrm{A}}$ in Fig. 4, which is zero at a time slightly less than $334.5 \mathrm{~h}$ MET. At this time the aerodynamic acceleration must be zero and any value other than zero allows for a refinement of the instrument bias for this axis.

\section{Data Processing}

A brief overview of the data processing required to arrive at the aerodynamic signals is given for completeness. A more in-depth description is given in reference 5. After the mission, the OARE reentry file is downloaded from the OARE memory in a raw digital format which provides the OARE measurements in a coded bit structure. This raw flight data file is processed first in order to transform the flight data into useful quantities such as the accelerations (in counts) along the $\mathrm{X}$-, $\mathrm{Y}$-, and $\mathrm{Z}$-axes along with numerous other quantities such as MET time, gimbal angles, the range code of each axis, bias and scale factor calibration flags, various temperatures, etc. The details of the raw digital format are given in reference 9 . The acceleration data rate is 10 samples/second.

\section{Calibration Factors}

Bias and scale-factor ratio measurements are used to produce the best estimates of the $\mathrm{X}, \mathrm{Y}$, and $\mathrm{Z}$-axis absolute accelerations at the OARE sensor location. Bias and scale factor measurements are made by the OARE throughout the entire mission. The entire mission calibration data set provides insight into the validity of the calibration process. This procedure also provides estimates of the errors associated with generating absolute accelerations which are necessary for producing reliable aerodynamic accelerations, particularly at the nano-g level. The details on how these measurements are made and interpreted have been described in the literature ${ }^{5}$ and will not be repeated here.
The bias and scale factor data throughout the entire STS-62 mission have been carefully examined and the results reported in reference 10 . This ensemble of flight data has been used to obtain the values applicable for the reentry phase for this mission. Meaningful biases are determined using the calibration data just prior to the reentry mode initiation between approximately 0.6 and 20.4 hours prior to deorbit burn initiation. During this interval six calibration points are examined, but only two are reliable due to the maneuvering done by the Orbiter to orient itself in preparation for deorbit. The bias value results are given in Table 1 .

The scale factors tend to remain constant throughout the flight. The in-flight scale factors measurements are divided into four categories; (1) high rate, normal direction, (2) high rate, reverse direction, (3) low rate, normal direction, and (4) low rate, reverse direction. High and low outliers are eliminated from each calibration data set and then the averaged scale factor is calculated from each set. Then, the average of all four sets is found and is given in Table 2, except for the $C$-range, $Z$-axis values.

The scale factors for the $C$ range, $Z$ axis are found to be unreliable for all OARE flights to date. To circumvent this problem, the scale factor calibration deduced from the Orbiter rotation about the center-of-gravity have been used. An equivalent scale factor effect can be generated by holding the sensor motionless and rotating the spacecraft. This has been accomplished successfully on past Orbiter missions and scale factor calibration values from Orbiter maneuvers compare well with scale factor results generated from the OARE calibration station, when reliable data is obtained 4 . Thus, there is reason to believe that the $Z$-axis scale factor determined by the flight maneuver is applicable since the maneuver data closely match the other two axes. The scale factor used in this analysis was the value determined during maneuvers on the STS-58 mission. The scale factor calibration values used in the analysis are given in Table 1.

Table 1. STS-62 Reentry Calibration Factors

\begin{tabular}{|c|c|c|c|}
\hline Range & Axis & Bias, ug & $\begin{array}{c}\text { Scale } \\
\text { Factor }\end{array}$ \\
\hline A & $\mathrm{X}$ & -6.638 & 1.03 \\
\hline $\mathrm{A}$ & $\mathrm{Y}$ & +1.144 & 1.12 \\
\hline $\mathrm{A}$ & $\mathrm{Z}$ & -136.566 & 1.09 \\
\hline $\mathrm{B}$ & $\mathrm{X}$ & -0.099 & 1.02 \\
\hline $\mathrm{B}$ & $\mathrm{Y}$ & +0.721 & 1.13 \\
\hline $\mathrm{B}$ & $\mathrm{Z}$ & -14.158 & 1.11 \\
\hline $\mathrm{C}$ & $\mathrm{X}$ & +0.048 & 1.01 \\
\hline $\mathrm{C}$ & $\mathrm{Y}$ & +0.737 & 1.14 \\
\hline $\mathrm{C}$ & $\mathrm{Z}$ & -7.288 & 1.08 \\
\hline
\end{tabular}

\section{Model Corrections}

The calibrated low-frequency OARE accelerations contain signals other than aerodynamic, such as rotational effects due to the sensor not being on the center-of-gravity, and gravity-gradient effects, both in-plane and out-of-plane. Models are applied in order to remove these effects from the acceleration measurements. The application of the models require information that is obtained from NASA-JSC in the 
form of MSID's. Specifically, the parameters required for any given time are: position, body quaternions (i.e. body orientation), velocity, and $\mathrm{p}, \mathrm{q}$, and $\mathrm{r}$ rotation rates about the Shuttle's X, Y, and Z body axes. With these data, gravitygradient, out-of-plane, and rotational acceleration components are calculated for each body axis from the Orbiter center-ofgravity to the location of the OARE sensor. The total correction to each axis is given in Fig. 5.

\section{Further Data Adjustments}

The residual acceleration found at the Orbiter center-ofgravity would be solely due to aerodynamic forces on the Orbiter if the correction models and the measurement inputs were perfect, the biases and scale-factor ratios were exact, and there were no other external forces on the Orbiter. Of course, this is not the case and the data must undergo some adjustments in order to extract the aerodynamic accelerations.

Significant forces on the Orbiter are generated on a continuing basis by the Orbiter's APUs (Auxiliary Power Units) during reentry and must be removed. These 3 units produce pulses of gas which rotate turbines and are used to provide the necessary power to move the control surfaces during reentry. The APU gases are ejected from ports at the base of the vertical tail and induce accelerations mainly in the z-body-axis direction. In flight, one of these units is activated prior to deorbit burn. The other two are activated nearly simultaneously after deorbit burn and prior to the entry attitude orientation of 40 degs. It was found that their effect on the $\mathrm{X}_{\mathrm{b}}$-axis is insignificant, but is considerable for the

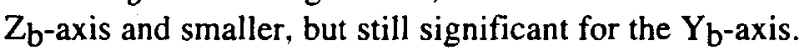

The $\mathrm{Z}_{\mathrm{b}}$-and $\mathrm{Y}_{\mathrm{b}}$-axis data with thruster activity about the time when the first APU is initiated can be seen in Figures 6 and 7. The adjustments made for the first APU initiation upon the $\mathrm{Z}_{\mathrm{b}}$-and $\mathrm{Y}_{\mathrm{b} \text {-axes accelerations can also be }}$ seen in these figures. Averages of the accelerations before and after APU initiation are performed and the difference taken. The adjustments are $13.382 \mu \mathrm{g}$ for the $\mathrm{Z}_{\mathrm{b}}$-axis and $2.953 \mu \mathrm{g}$ for the $\mathrm{Y}_{\mathrm{b}}$-axis. These adjustment values are subtracted from the data set after the initiation of the first APU.

\section{Further Bias Refinements}

During the Orbiter reentry stage, which begins several hours before deorbit burn, the Orbiter has more thruster activity in preparation for reentry (as opposed to the orbital phase of the mission) and the payload bay doors are closed which necessitates a more active flash evaporator system (FES). These additional small forces generated by the Orbiter are not modeled, and, in addition, the removal of the first APU effect is not perfect, thus resulting in a residual acceleration offset. These small adjustments (or zero offsets) can be found for any given axis when the aerodynamic forces are theoretically zero. Such is the case for the $\mathrm{X}_{\mathrm{b}}$ body axis when the angle-of-attack $\alpha$, is 90 degs. Similarly, the aerodynamic forces in the $\mathrm{Z}_{\mathrm{b}}$-direction disappear when $\alpha$ is 0 or 180 degs. These adjustments are made by effecting an average of the data near these specific points (i.e. finding a smooth curve which passes through the data on both sides of the point under investigation), and then subtracting this average from the entire data set for the given axis.

The mission time at which $\alpha=180$ degs. occurs after the initiation of the first APU, at around $334.380 \mathrm{~h}$ MET. The respective adjustments to produce zero accelerations at this time in the $\mathrm{Z}_{\mathrm{b}}$-axes is $3.017 \mu \mathrm{g}$.

\section{Second \& Third APU Effect}

Adjustments are also required when the additional two APU's are activated. These adjustments are found to be $19.006 \mu \mathrm{g}$ for the $\mathrm{Z}_{\mathrm{b}}$-axis and $6.068 \mu \mathrm{g}$ for the $\mathrm{Y}_{\mathrm{b}}$-axis. The combined effect of the first APU and of APU- 2 and 3 are subtracted from the data subsequent to about $334.54 \mathrm{~h}$ MET.

\section{Further Bias Checks}

Removing the effect of the APU's from the data requires an averaging process. In general, experience with averaging techniques has been reliable on past missions, but for this flight the actual turn-on of the remaining APU's was done while the OARE was performing sensor bias calibrations. This introduced more error in the process since the data which represented the signal prior to the second and third APU turn-on is more remote (in time) than in past missions. Thus, it is not likely that the second and third APU effect have been completely removed from the data. However, one additional check can be performed on the $\mathrm{Z}_{\mathrm{b}^{-}}$ axis data, after all three APU's have been activated, at MET = 334.575 hours. At this time, $\alpha=0$, and therefore the residual acceleration along the $\mathrm{Z}_{\mathrm{b}}$-axis at the Orbiter centerof-gravity should be zero. The residual acceleration is found to be $2.08 \mu \mathrm{g}$. This is subtracted from the subsequent $\mathrm{Z}_{\mathrm{b}}$ axis data.

\section{Reentry Measurements}

\section{Body-axis Components}

The aerodynamic accelerations for the $\mathrm{X}_{\mathrm{b}}$ - and $\mathrm{Z}_{\mathrm{b}}$ directions in the most sensitive $C$ range are shown in Fig. 8 as a function of mission elapsesd time. Each of these acceleration channels are represented by a 50 point moving average, to be used later in forming ratios. The accelerations are extended to lower altitudes by including the two other OARE ranges, namely the B- and A-range data (Figure 9). Below about $334.66 \mathrm{~h} \mathrm{MET}$, both $\mathrm{A}_{\mathrm{xb}}$ and $\mathrm{A}_{\mathrm{zb}}$ measurements are relatively flat on a liner scale and, as will subsequently be seen on a later graph, lie in the freemolecule-flow regime. To the right of the time $344.660 \mathrm{~h}$, the onset of the transition regime begins, and both axis begin to become appreciable larger as the Orbiter travels deeper into the atmosphere with $A_{z b}$ becoming larger faster than $A_{x b}$, as expected. The jump in the accelerations at about $334.665 \mathrm{~h}$ is due to an abrupt change in the angle-of-attack, which shows mostly in the $A_{z b}$ channel. 


\section{Normal to Axial Ratios}

Acceleration ratios are the only way to obtain direct measurements of aerodynamic coefficients from accelerometers since the dynamic pressure cancels. The ratio $A_{Z} / A_{X}$ is calculated for each OARE range and presented in Figs. 10 and 11 as a function of MET. The corresponding Orbiter orientation, $\alpha$, and altitude is also shown in the upper graph on each figure. Also shown in each figure is the free-molecule-flow prediction of $\mathrm{CN}_{\mathrm{N}} / \mathrm{C}_{\mathrm{A}}$ generated using the preflight Orbiter data base, presented earlier in Fig. 4. Departure from the theoretical predictions occurs at lower altitudes as the vehicle transitions from free-molecule-flow towards the hypersonic continuum. The departure of $A_{Z} / A_{X}$ from free-molecule-flow predictions is apparent at about $334.68 \mathrm{~h} \mathrm{MET} \mathrm{(Fig.} 10$ ), corresponding to an altitude of about $160 \mathrm{~km}$. These are the first acceleration measurements which clearly show the initial departure of the Orbiter from the free-molecule-flow regime. The approximate Knudsen numbers are discussed later, along with the numerical simulation comparisons.

At altitudes above about $210 \mathrm{~km}$, when the vehicle is at large negative angles-of-attack, the comparison with the predictions depart as the angle becomes more negative. Starting from about 25 degs. angle-of-attack to about -45 degs. the ratio measurements are noticeably smaller than the predictions. The difference is significant even though the measurements contain a lot of noise due to thruster activity in changing the angle-of-attack. This departure may be due in part to gases being injected into the forward upstream portion of the flow-field by both the APU's and the FES (the FES predominately expels water vapor through fuselage ports over the rear top wing area). As the vehicle pulls its nose up into the flow, and the density increases as altitude decreases, the effect disappears and the predictions match the measurements quite well.

Fig. 11, shows a dramatic departure between the measurement ratios and the theoretical free-molecule-flow predictions at lower altitudes, as expected. Earlier work using the Orbiter's IMU acceleration data showed (Fig. 3, ref. 10) $\mathrm{C}_{\mathrm{N}} / \mathrm{C}_{\mathrm{A}}$ approaches values of about 20 in the hypersonic continuum.

\section{Comparison of OARE and HiRAP Measurements}

The OARE results extend earlier measurements with HiRAP, which to date, has flown 16 flights on the Challenger and Columbia vehicles $6,12,13$. HiRAP, unlike OARE, is a gas damped pendulous accelerometer which cannot be readily calibrated on orbit, and has a resolution of about $1 \mu \mathrm{g}$ in each of its three axes. Because of these limitations, the HiRAP measurements do not reliably extend in altitude above about $140 \mathrm{~km}$. In contrast, the OARE measurements presented in this paper extend the measurements to orbital altitudes, well beyond the HiRAP capabilities.

On STS-62 both rarefied-flow accelerometer experiments were fully operational and provide an opportunity to compare directly results from both sets of flight equipment. The technique used to calibrate the HiRAP is well documented 12.13 and involves establishing a bias correction when the Orbiter is at an altitude which provides an input signal slightly less than the resolution of the sensors $(-1 \mu \mathrm{g})$. In addition, the bias is meticulously adjusted for slight temperature variations as measured by internal temperature sensors. HiRAP data collected on STS-62 (details of analysis to be reported separately) are shown in Fig. 12 along with OARE data to provide a direct comparison between the two experiments. These data are in good agreement at times greater than about $334.75 \mathrm{~h} \mathrm{MET,}$ which corresponds to altitudes below about $120 \mathrm{~km}$. At times less than $334.75 \mathrm{~h} \mathrm{MET}$, i.e. greater altitudes, the comparison is about as expected considering the limitations of the HiRAP sensors. OARE extends deeper into the atmosphere than HiRAP, mostly because of initial bias offsets in the HiRAP sensors.

\section{Comparisons of DSMC Predictions to Flight Measurements}

\section{Initial Environment Estimate}

To make comparisons between flight data and predictions via numerical simulation techniques requires knowledge of the environment under which the flight data were collected. It is not sufficient to use vehicle position, e.g. altitude, and then revert to a model atmosphere to generate the gas properties. Invariably, the match between the numerical simulation and the flight data will be unsatisfactory due to variances associated between atmosphere models and real-world environment. To circumvent this problem, it is possible to generate a satisfactory estimate of the atmosphere properties during the time-of-flight by using the normal accelerometer channel (i.e. $A_{z b}$ ) for this vehicle. The reason for this approach is illustrated in Fig. 13 which shows the free-molecule-flow $\mathrm{C}_{\mathrm{N}}$ generated from the STS62 trajectory parameters (i.e., $\alpha$ and $\beta$ ) as a function of time at the fringes of the rarefied-flow transition regime. Superimposed on the graph is the best estimate of the $\mathrm{C}_{\mathrm{N}}$ (= 1.23) for the hypersonic continuum from reference 14 (Fig. 7, p.359). As shown, the continuum value is at most about 17 percent lower than the free-molecule-flow values for the near fixed angle-of-attack flown by the Orbiter. There is some variation because the values shown account for the Orbiter's actual angle-of-attack which varies a little (typically less than $1.0 \mathrm{deg}$.) during this portion of the flight. There are also differences in aerodynamic-surface settings; in flight body flap deflection $=11.7 \mathrm{deg}$. and elevon deflection $=2.1$ deg., while the continuum body flap deflection $=16 \mathrm{deg}$. and there is no elevon deflection.

Consider the force equation;

$$
\rho=\frac{2 A_{z b}}{v_{a}^{2}\left(-C_{N}\right)\left(\frac{s}{m}\right)}
$$

where $A_{z b}$ is the normal axis aerodynamic acceleration measurements, $\mathrm{V}_{\mathrm{a}}$ is the air relative velocity, $(\mathrm{S} / \mathrm{m})$ is the Orbiter reference area $\left(S=249.909 \mathrm{~m}^{2}\right)$ to mass ratio, and $C_{N}$ 
is the normal axis force coefficient. All quantities are known on the right hand side of the equation except $\mathrm{C}_{\mathrm{N}}$. If the freemolecule-flow normal coefficient is used in the calculation of density, then the error incurred by this assumption is at most about 17 percent. This amount of error occurs when the vehicle resides in the hypersonic continuum regime. At higher altitudes, corresponding to earlier flight times, the error becomes progressively smaller, becoming negligible in the free-molecule-flow regime. Since the free-molecule-flow $\mathrm{C}_{\mathrm{N}}$ value used is larger than the correct value, the density will be too small relative to the actual density value, but sufficiently accurate to obtain an initial first-order estimate of the flight environment. Second order adjustments can be made after the first iteration, using the numerical simulation value of $\mathrm{C}_{\mathrm{N}}$. For this report, the second order adjustments are not warranted since only the fringes of the rarefied-flow transitional regime are examined.

The corresponding variation of density with altitude using the free-molecule-flow $\mathrm{CN}_{\mathrm{N}}$ is shown in Fig. 14. Included in this figure is the $1976 \mathrm{U}$. S. standard atmospheric model 15 as a reference. The density variation from the present analysis is somewhat different from the model atmosphere, but not as much as expected. The flight data is slightly lower (15-20\%) than the model at altitudes less than about $105 \mathrm{~km}$. This is expected since the free-molecule-flow $\mathrm{C}_{\mathrm{N}}$ is used in the calculation which provides an underestimate of the density, as discussed earlier. Above about $170 \mathrm{~km}$ there is significant departure from the model atmosphere. This is probably a real difference, since the acceleration ratio matches well with the predicted coefficients at these altitudes (see Fig. 10).

The agreement between the density inferred from the flight data and the model atmosphere density shown in Fig. 14 is remarkably good. However, past flights have exhibited significant departures from atmosphere models 16 . The point here is that comparison of flight data to simulations must proceed by first establishing the flight environment/model relationships used by DSMC simulations, and density is the key parameter.

\section{DSMC Results}

Results from three different DSMC codes are presented in this paper, namely Rault ${ }^{17}$, and the DAC and G3 codes used by the authors. The results of Rault were computed using a three dimensional direct simulation Monte Carlo program which contains an unstructured Cartesian grid. The results denoted " $\mathrm{G} 3$ " were computed using an unstructured body-fitted tetrahedral grid code and the results labeled "DAC" used a structured Cartesian grid code with an embedded grid refinement. The latter two codes are described in reference 18. All three DSMC codes use similar physical models for describing intermolecular collisions and gas-surface interactions.

Fig. 15 shows a comparison between the flight density derived values using the free-molecule-flow $\mathrm{C}_{\mathrm{N}}$ and the corresponding density values used in the DSMC simulations. The density comparison for both the DAC and G3 codes are included on Fig. 15 for reference, since input gas properties from the 1976 U.S. standard model are used for these codes.
However, Rault data is existing data and the density used in his calculations must be compared before a viable comparison with flight data can be made. In general, the comparison is excellent except for the lowest and highest altitude data points of Rault. The highest altitude point (170 $\mathrm{km}$ ) would require a slight shift of about 1 to $2 \mathrm{~km}$ lower to match the flight conditions. The lowest $(100 \mathrm{~km})$ altitude point difference is explained by the fact that no iteration has been performed to adjust the flight density using the transitional-flow $\mathrm{C}_{\mathrm{N}}$ as opposed to the free-molecule-flow $\mathrm{C}_{\mathrm{N}}$ as discussed above. In effect, using the smaller $\mathrm{C}_{\mathrm{N}}$ would shift the flight density closer to the model and closer to Rault's simulation conditions.

Fig. 16(b) illustrates the anticipated behavior of the normal force coefficient, $\mathrm{C}_{\mathrm{N}}$ as a function of altitude and Fig. 16(a) shows a corresponding approximate free-stream Knudsen Number calculation using the 1976 U.S. standard atmospheric model. The Knudsen Number is the ratio of mean-free path (average molecule collision distance) to a reference length and is often used in the literature as a flow regime indicator; large values indicate free-molecule-flow regime, small values indicate the hypersonic continuum flow regime. The mean free path calculation for the 1976 model assumes a solid hard sphere molecular diameter $\left(3.65 \times 10^{-10}\right.$ $\mathrm{m}$ ) and the model density and mean molecular weights. Two reference lengths are used, namely the Orbiter body length $(32.7736 \mathrm{~m}$ ) and the Orbiter mean aerodynamic chord length $(12.0609 \mathrm{~m})$. Based upon typical interpretation of $\mathrm{Kn}$ as a flow-regime indicator; at $90 \mathrm{~km}$ altitude $\left(\mathrm{Kn} \sim 10^{-3}\right)$, the vehicle is near or in the continuum regime, while at $190 \mathrm{~km}$ $(\mathrm{Kn} \sim 10)$ the vehicle is in the free-molecule-flow regime. At about $140 \mathrm{~km}(\mathrm{Kn} \sim 1)$ the transition is taking place where the vehicle resides in the rarefied-flow transition regime, which is neither fully continuum nor free-molecule-flow.

The lower graph shows the free-molecule-flow $\mathrm{CN}_{\mathrm{N}}$ behavior as a function of altitude, using the Orbiter data book values presented earlier on Fig. 4. The $\mathrm{C}_{\mathrm{N}}$ data take into account the as-flown orientation of the Orbiter. The influence of the angle of attack on this coefficient is readily seen, particularly above $170 \mathrm{~km}$. Included are the results of the three DSMC calculations for this coefficient. The work of Rault uses a fixed angle-of-attack of $40 \mathrm{deg}$., while the current authors use the flight values, although the differences in angle from $40 \mathrm{deg}$. are small $( \pm 0.6 \mathrm{deg}$.). An approximate behavior of the $\mathrm{C}_{\mathrm{N}}$ is generated using a leastsquare quadratic fit to the eight DSMC data values below 140 $\mathrm{km}$ and the result is shown in Fig. 16. This directly illustrates the approximate error introduced in the density calculation presented earlier. A first order correction required to adjust for the transition coefficient is about 12 percent larger density at $92 \mathrm{~km}$ reducing to zero at about $140 \mathrm{~km}$. This results in moving the curve to slightly larger values than those shown on Fig. 15, but hardly noticeable for the chosen graph scale.

As stated earlier, the density issue could be eliminated by looking at the ratio of accelerations, since all parameters, including density, cancel except for the coefficients. That is,

$$
\frac{A_{Z}}{A_{X}}=\frac{C_{N}}{C_{A}}
$$


However, this is not entirely the case, since the ratio data from flight (measured in the time domain) must be placed in the spatial domain used by the numerical codes. Valid comparison of the flight and DSMC data requires the densities, or Knudsen number, or equivalent altitudes for a given density to match. For this tlight, there is a good match between the tlight derived density and the numerical simulations so that the force coefficient ratio data can be compared directly with relatively little error. Fig. 17 shows a comparison of the flight values of normal-to-axial coefficient ratio to predictions via the three numerical simulations. Also shown on the graph is the Orbiter data book predictions for the free-molecule-flow conditions. Seen again is the departure from free-molecule-flow which occurs at roughly $160 \mathrm{~km}$. Referring to Fig. 16(a) this corresponds to a Kn between 2 to 6 depending upon reference length. There is excellent agreement between the flight values and all three numerical simulations. There is a slight trend to underpredict the $\mathrm{C}_{\mathrm{N}} / \mathrm{C}_{\mathrm{A}}$ ratios the deeper the vehicle goes into transition. Possible explanations are the neglect of . chemistry in the simulations and inadequate cell resolution. However, overall comparisons are within about 5 percent of the flight data at all altitudes simulated.

\section{Summary}

OARE reentry data have been successfully recorded for the first time during the STS-62 mission from orbital altitudes down to approximately $90 \mathrm{~km}$. The Orbiter's reentry aerodynamic acceleration signals were extracted by using biases and scale-factor measurements which were recorded during the orbital part of this mission, in conjunction with ancillary flight data on position, body orientation, velocity, and rotation used to transform accelerations to the Orbiter's center-of-gravity. Unmodeled accelerations, such as the effects of APU's have been removed from the data and aerodynamic acceleration offsets have been refined using conditions when the sensor input axis is perpendicular to the flow. The resultant aerodynamic acceleration flight data from OARE (a nano-g sensor) on STS-62 compares very well with another independent microgravity flight experiment called HiRAP (a micro-g sensor), which also was operational on the flight. This agreement further validates the OARE aerodynamic signal extraction transformation procedures since there is an excellent match with the data from the more mature HiRAP experiment. Ratios of measured aerodynamic accelerations $\left(\mathrm{A}_{\mathrm{z}} / \mathrm{A}_{\mathrm{x}}\right)$ were formulated throughout the entire upper-altitude reentry phase. Comparisons between the flight data and theoretical Shuttle Orbiter data book values of $\mathrm{C}_{\mathrm{N}} / \mathrm{C}_{\mathrm{A}}$ in free-molecular-flow show generally good agreement. The departure from freemolecule-flow to transition into the hypersonic continuum regime is readily observed for the first time in the OARE reentry data and takes place at about $160 \mathrm{~km}$. This corresponds to an approximate $\mathrm{Kn}$ of between 2 to 6 depending upon reference length. In addition, in the freemolecule-flow regime, while the vehicle is at negative angles-of-attack during reentry, the free-molecule-flow predictions do not match the measurements. This mismatch appears to correlate with angle-of-attack and altitude. It is surmised that a part of this prediction mismatch is a result of gases emanating from the Orbiter interacting with the forward flow-field. A more detailed investigation is, however, required to resolve this issue.

An estimate of the corresponding density environment for the acceleration measurement domain using the Orbiter's free-molecule-flow coefficient, $\mathrm{C}_{\mathrm{N}}$, provided a means to correlate with previous DMSC numerical simulations and provided a means to obtain atmosphere inputs for additional DSMC simulations using two different codes. The more recent DSMC calculations use the 1976 U. S. standard atmospheric model, which matches unusually well with the flight derived density on this mission. An examination of the DSMC simulations of the $\mathrm{C}_{\mathrm{N}}$ behavior as a function of altitude in the transition regime provides an estimate of the first order correction required to adjust the flight derived densities for transitional flow effects. The correction to $\mathrm{C}_{\mathrm{N}}$ is about 12 percent at $92 \mathrm{~km}$ and reduces to negligible amounts to altitudes above about $140 \mathrm{~km}$. There is excellent agreement (within 5\%) between the flight values of $A_{Z} / A_{X}$ and predictions via the three independent DSMC numerical simulation codes.

The OARE aerodynamic ratio data provided in this report represent a milestone for a winged orbiting reentry vehicle as it transitions from free-molecule-flow into the hypersonic continuum. Aerodynamic accelerations have been measured at much higher altitudes during reentry than previously obtained and excellent agreement has been obtained between predictions with detailed DSMC simulation codes and measurements. Ultimately, this benchmark data should provide enhanced understanding of the initial transition into the hypersonic continuum with direct benefits to future space transportation system development.

\section{References}

${ }^{1}$ Blanchard, R.C., Hendrix, M.K., Fox, J.C., Thomas, D.J., and, J.Y., "The Orbital Acceleration Research Experiment," Journal of Spacecraft and Rockets, vol. 24, no. 6, Nov.-Dec. 1987, pp. 504-511.

${ }^{2}$ Blanchard, R. C.; Nicholson, A. B.; and Ritter, J.: STS-40 Orbital Acceleration Research Experiment Flight Results During a Typical Sleep Period. International Journal for Microgravity Research and Applications, V/2 1992, pp. 86-93.

${ }^{3}$ Blanchard, R. C.; Nicholson, J. Y.; and Ritter, J. R.: Absolute Acceleration Measurements on STS-50 from the Orbital Acceleration Research Experiment (OARE), International Journal of Microgravity Research and Applications, VII/1, 1994, pp. 60-67.

${ }^{4}$ Blanchard, R.C., Nicholson, J.Y., Ritter, J.R. and, Larman, K. T. "OARE Flight Maneuvers and Calibration Measurements on STS-58," NASA TM-109093, Apr. 1994. 
${ }^{5}$ Blanchard, R.C., and Nicholson, J.Y., "OARE Rarefied-Flow Reentry Measurements from the OARE on STS-62," NASA TM-110182, Jun. 1995.

${ }^{6}$ Blanchard, R.C.; and Rutherford, J.F.: The Shuttle Orbiter High Resolution Accelerometer Package Experiment: Preliminary Flight Results. Journal of Spacecraft and Rockets, vol. 22, no. 4 July-August 1985, p 474.

${ }^{7}$ Bate, R. B., Mueller, D. D., and White J. E., Fundamentals of Astrodynamics, 1971, Dover Publications, Inc.

8"Operational Aerodynamic Design Data Book," Rockwell International, Space Division, Downey, CA STS 85-0118 CHG 3, September 1991.

${ }^{9}$ Anon., "Orbital Acceleration Research Experiment Flight Software Requirements," Drawing No. 301018, Rev I, Canopus Systems, Inc., Ann Arbor, MI, Apr., 1988.

${ }^{10}$ Blanchard, R.C., and Nicholson, J.Y., "Summary of OARE Flight Calibration Measurements," NASA TM109159, Jan. 1995.

${ }^{11}$ Blanchard, R. C., Larman, K. T., and Moats C. D., "Rarefied-Flow Shuttle Aerodynamics Flight Model," Journal of Spacecraft and Rockets, vol. 31, no. 4, Jul.-Aug. 1994, pp. 550-556.

12Blanchard, R. C.; Larmen, K. T.; and Barrett, M.: The High Resolution Accelerometer Package (HiRAP) Flight Experiment Summary for the First Ten Flights, NASA RP1267, March 1992.

${ }^{13}$ Blanchard, R. C., Larman, K. T., and Moats C. D., "Flight Calibration Assessment of HiRAP Accelerometer Data," AIAA Paper 93-0836, January 1993.

${ }^{14}$ Weilmuenster, K.J., Gnoffo, P.A., and Greene, F.A., "Navier-Stokes Simulations f Orbiter Aerodynamic Characteristics Including Pitch Trim and Bodyflap," Journal of Spacecraft and Rockets, vol. 31, no. 3, May-Jun. 1994, pp. 355-366.

15 Anon., U.S. Standard Atmosphere, 1976, NOAA, NASA, and U.S. Air Force, Oct., 1976.

16 Blanchard, R. C.; Nicholson, J. Y.; Larman, K. T.; and Ozoroski, T. A., "Orbiter Rarefied-Flow Aerodynamics and Upper-Atmosphere Density Flight Measurements.", Proceedings of Orbiter Experiment (OEX)

Aerothermodynamics Symposium, Session 3, Williamsburg, VA, April 1993. NASA Conference Publication 3248, Part II, pp. 553, April 1995.

17 Rault, D.F.G., "Aerodynamics of the Shuttle Orbiter at High Altitudes," Journal of Spacecraft and Rockets, vol. 31, no. 6, Nov.-Dec. 1994, pp. 944-952.
${ }^{18}$ Wilmoth, R. G., LeBeau, G. J., and Carlson A. B., "DSMC Grid Methodologies for Computing Low-Density Hypersonic Flows About Reusable Launch Vehicles," AIAA Paper 96-1812, June 1996.

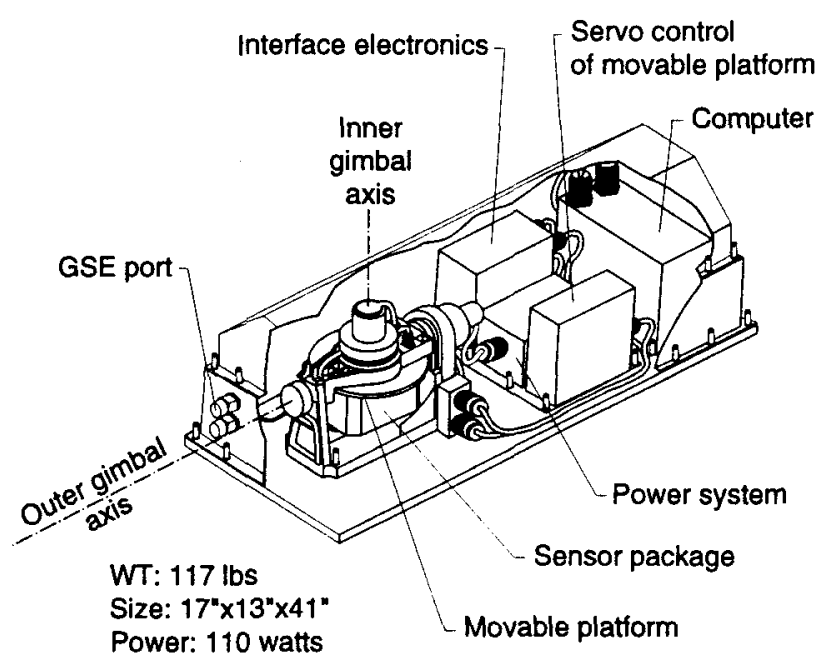

Fig. 1 OARE system layout.

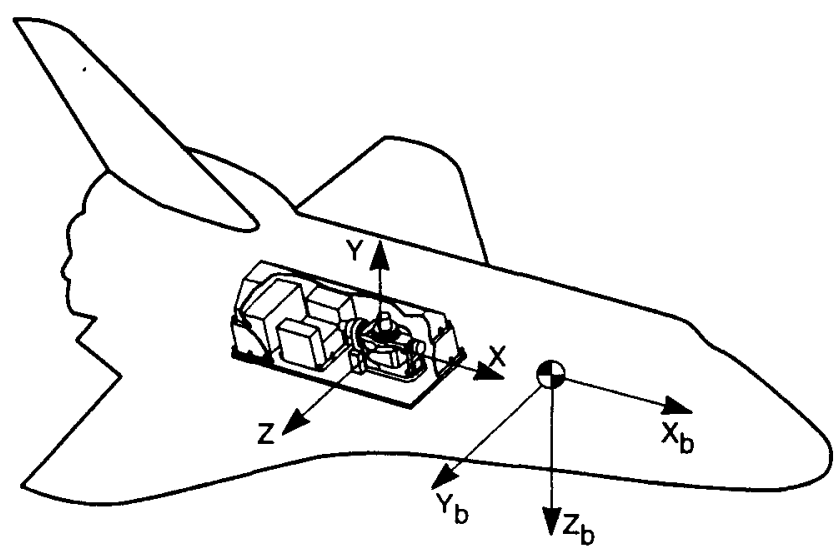

Fig. 2 OARE (X,Y,Z) and Orbiter body axes $\left(X_{b}, Y_{b}, Z_{b}\right)$ coordinate systems. 


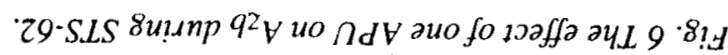

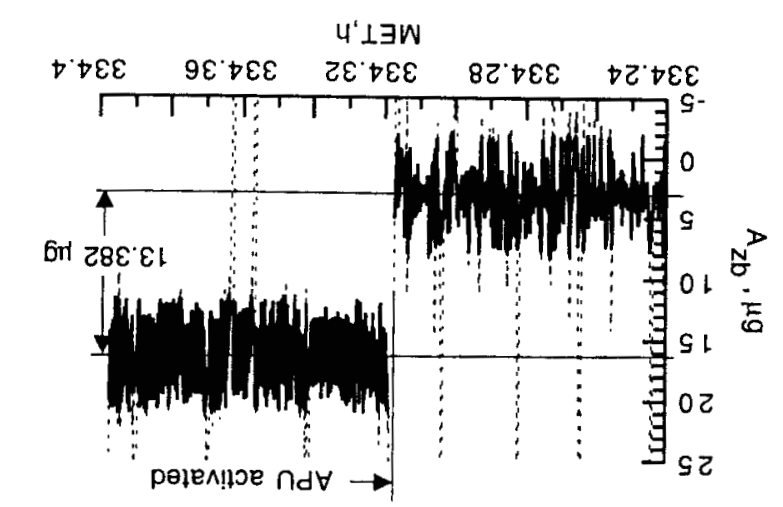

les ејер pәбелөл

los eృep 키미으

29-SIS $10 \mathrm{f}$

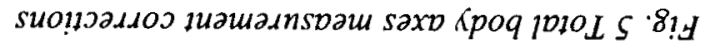

4'1 $1 \exists w$
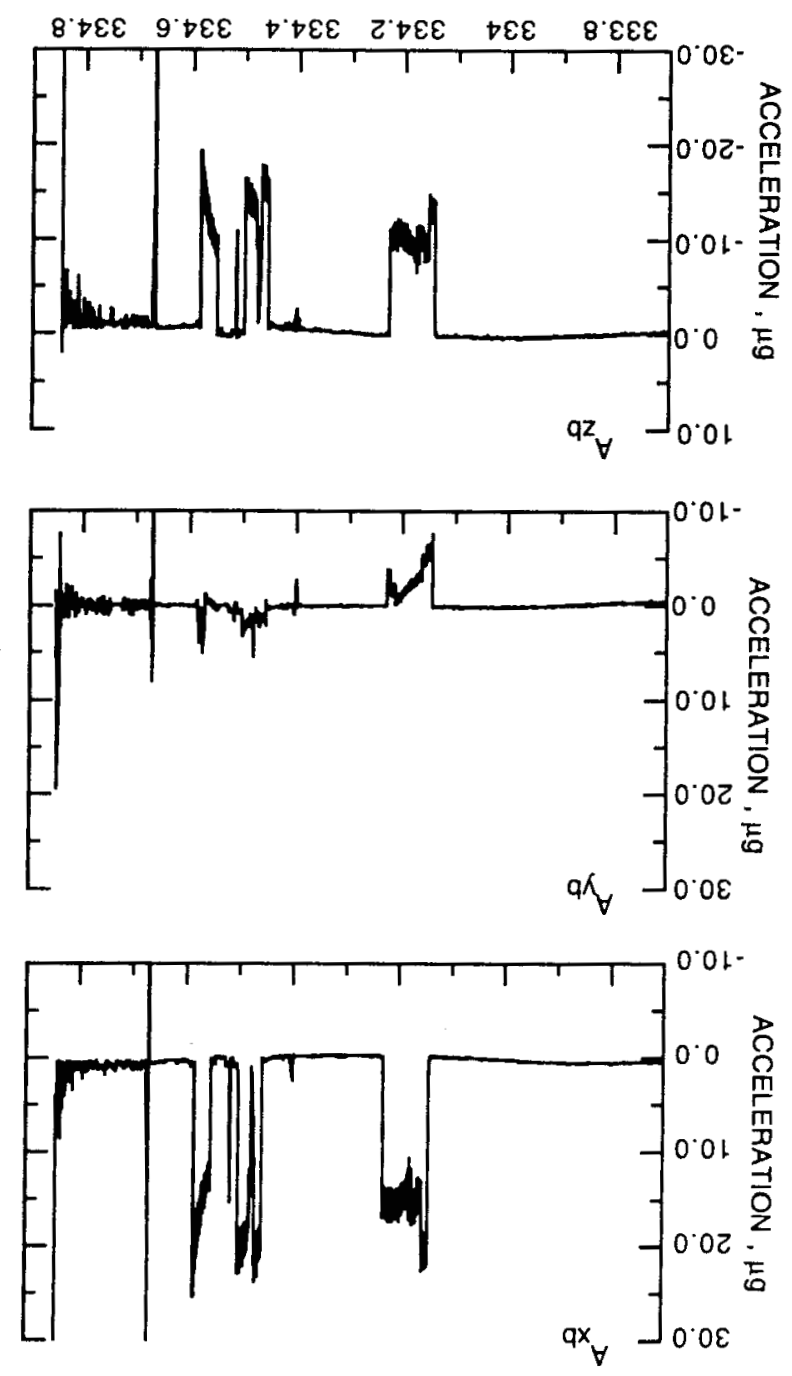

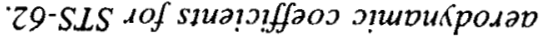

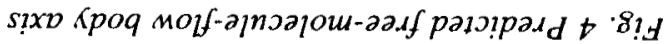

4 ' $\perp \exists W$

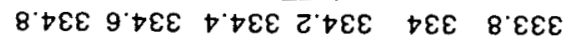

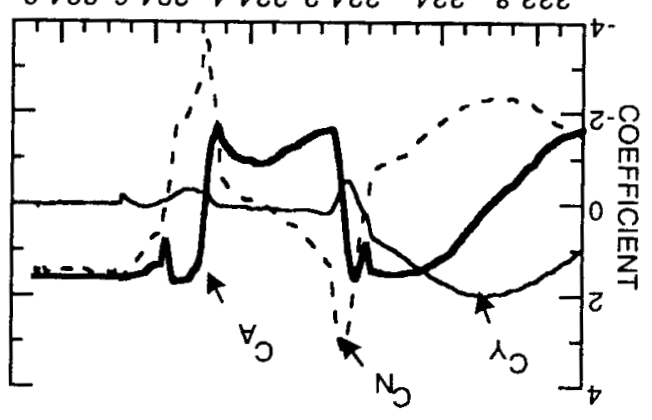

29-SLS $10 \mathrm{f}$

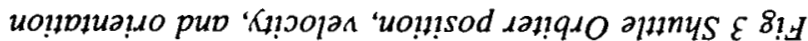

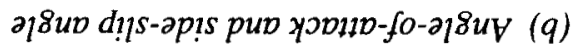

4 ' $\perp \exists W$

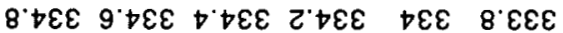

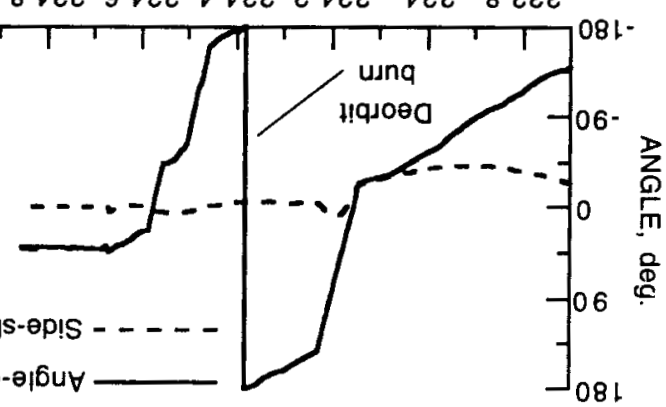

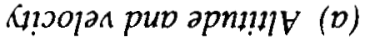

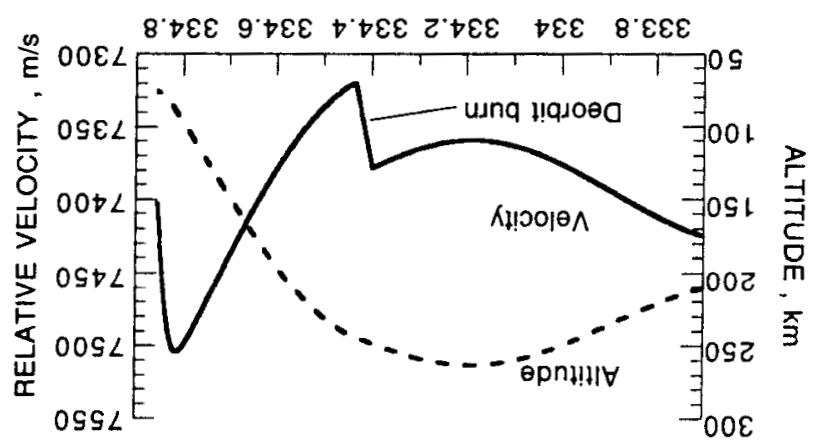




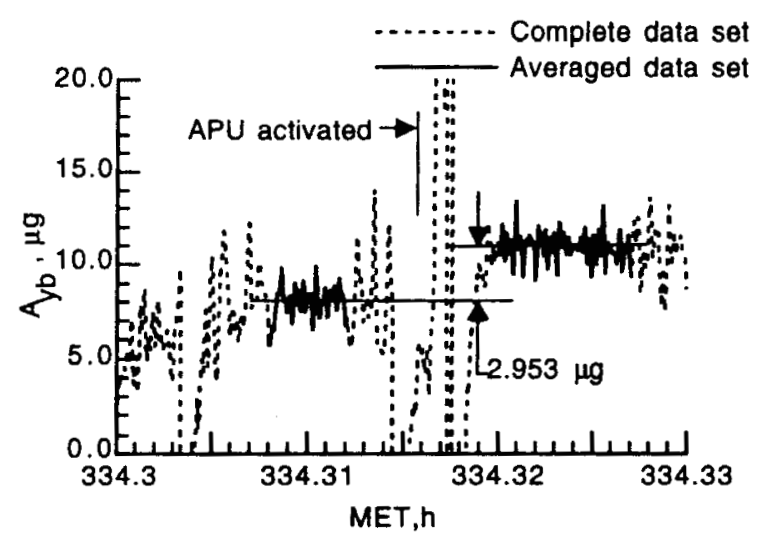

Fig. 7 The effect of one APU on $A_{y b}$ during STS-62.
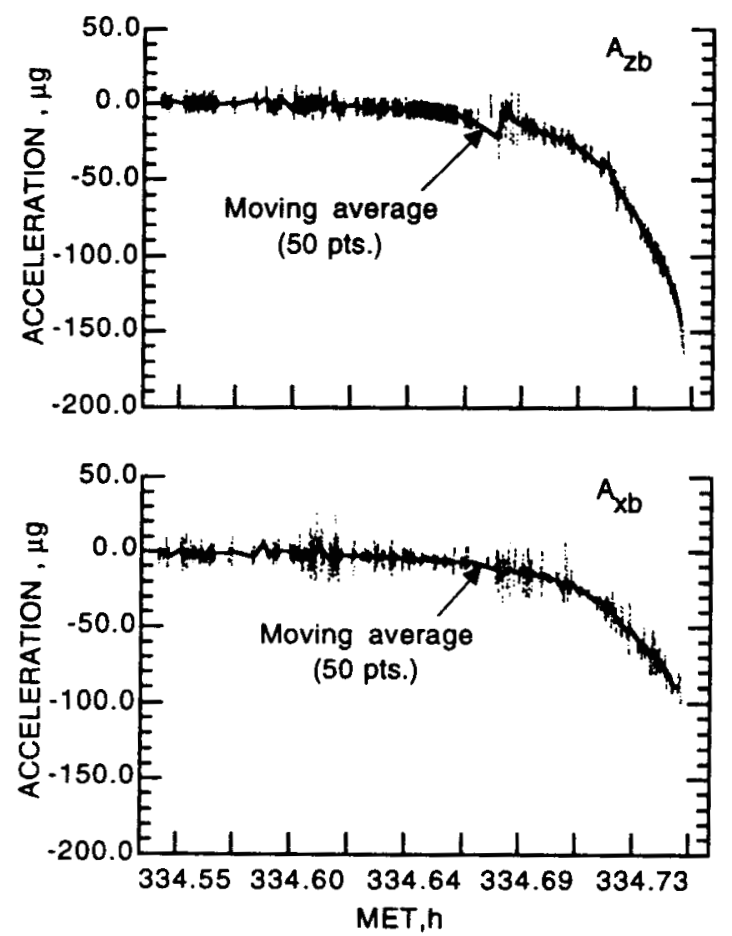

Fig 8 Normal and axial acceleration measurements at the onset of the free-molecule-flow transition regime (C-Range).

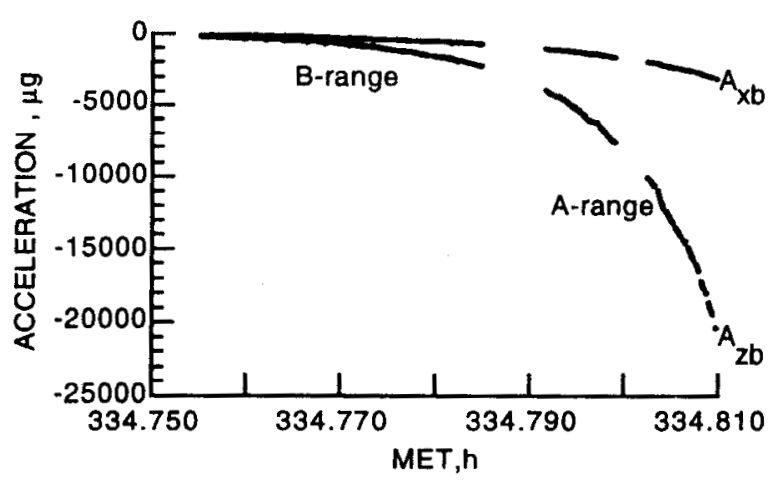

Fig. 9 Normal and axial measurements in the rarefiedflow transition regime.

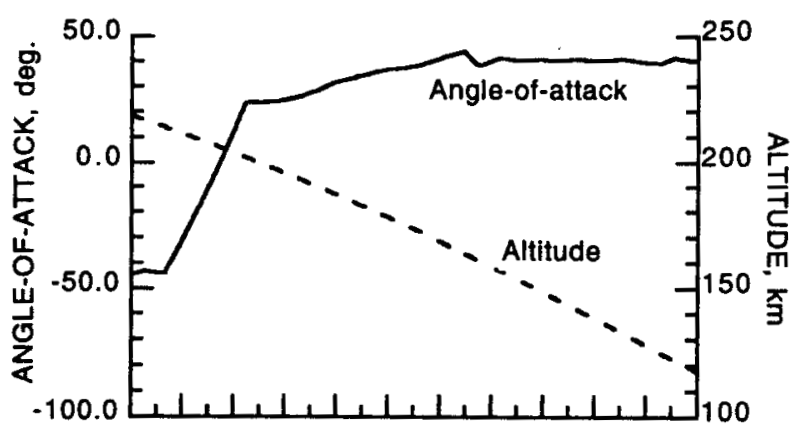

(a) Orbiter angle-of-attack and altitude

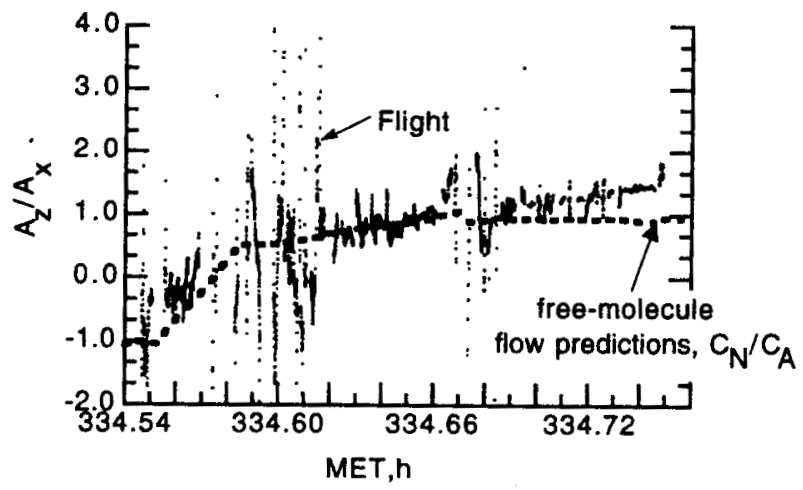

(b) OARE C-Range measurements

Fig 10 Normal-to-axial measurements at the onset of rarefied-flow transition regime. 


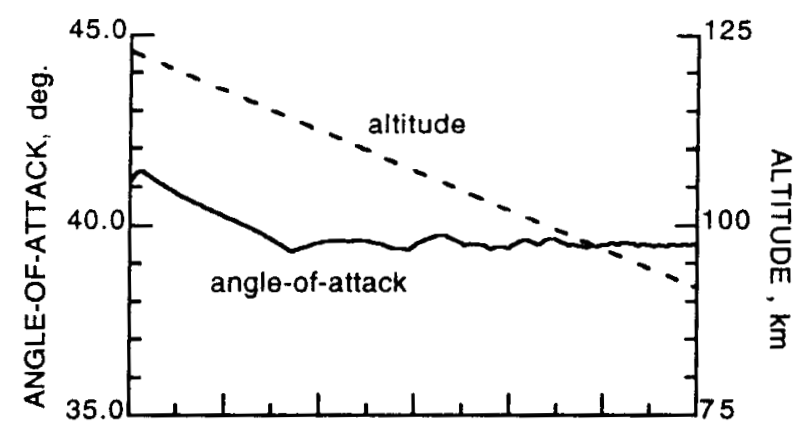

(a) Orbiter angle-of-attack and altitude

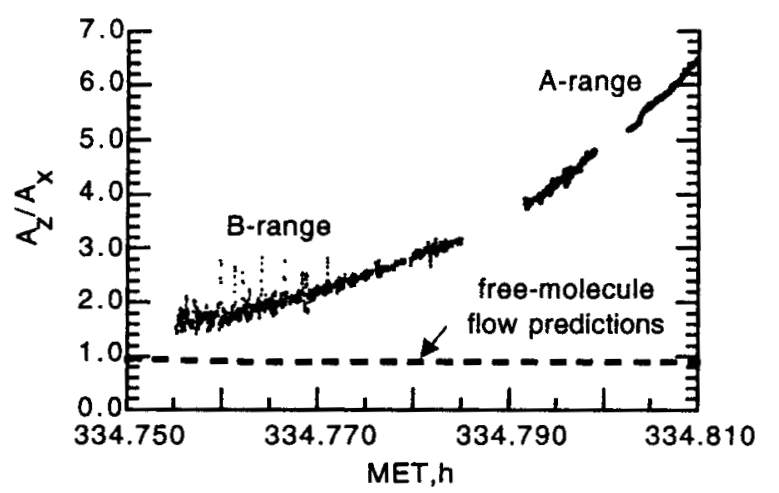

(b) OARE B-and A-Range measurements

Fig 11 Normal-to-axial measurements in the rarefied-flow transition regime.

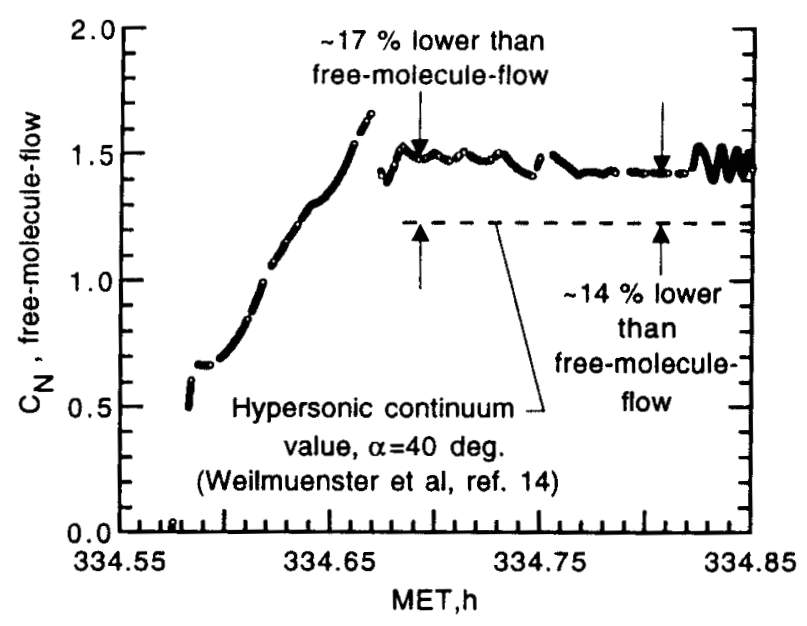

Fig 13 Comparison of $C_{N}$ values between free-moleculeflow and hypersonic continuum regime.

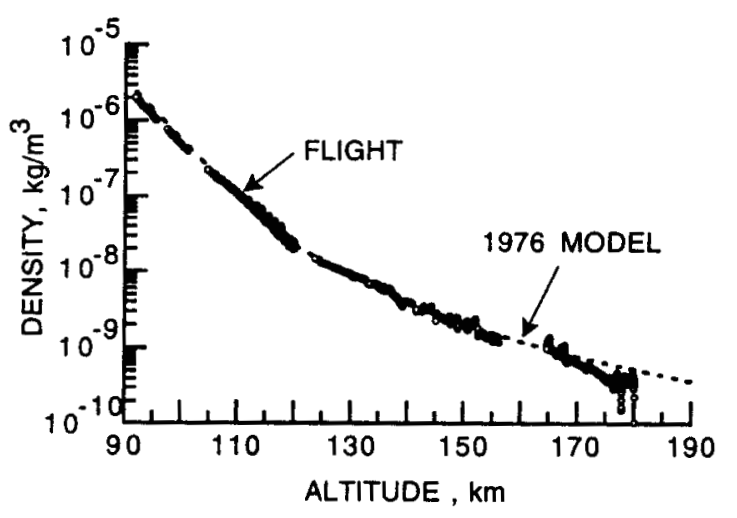

Fig 14 Comparison between 1976 U.S. standard model density and flight derived density using free-moleculeflow normal force coefficient, $C_{N}$.

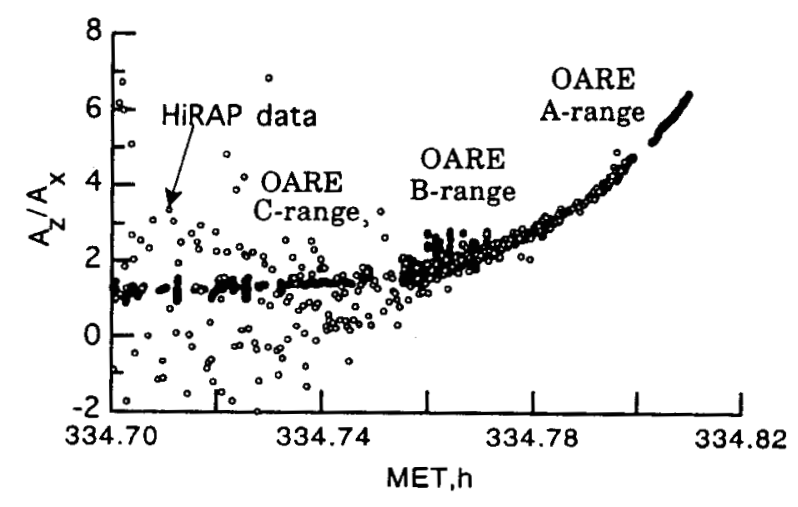

Fig 12 Comparison between OARE and HiRAP flight data on STS-62.

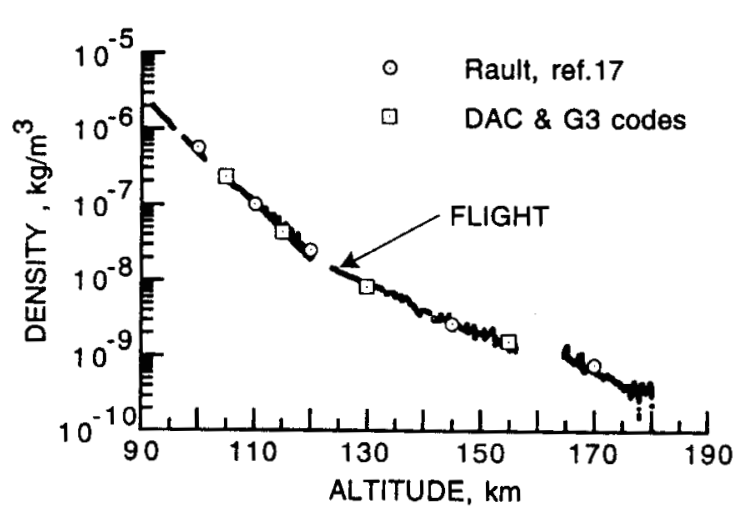

Fig 15 Values of density used in DSMC codes compared to flight derived density using free-molecule-flow coefficient, $C_{N}$. 


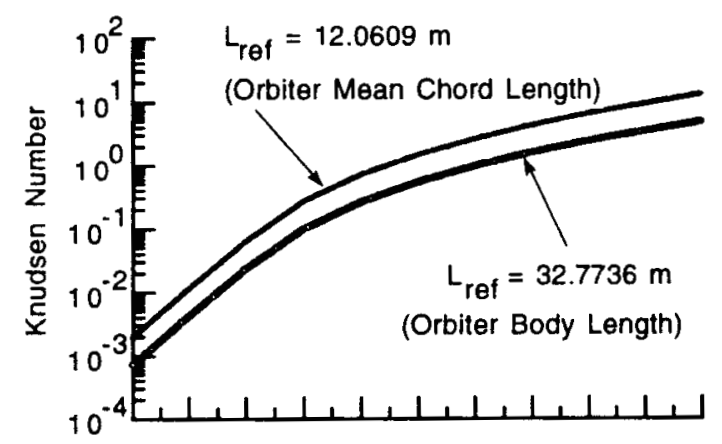

(a) Kn (using 1976 U.S. standard atmosphere model)

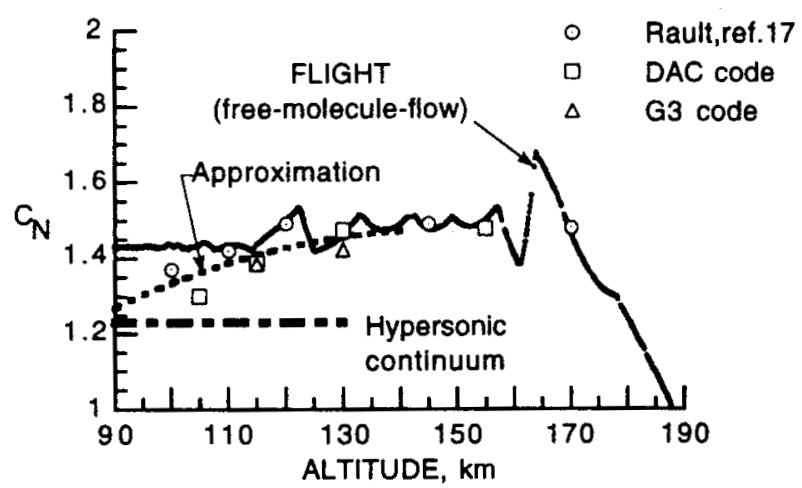

(b) normal force coefficents

Fig 16 Approximate Knudsen Number and normal force coefficient behavior in the rarefied-flow transition regime.

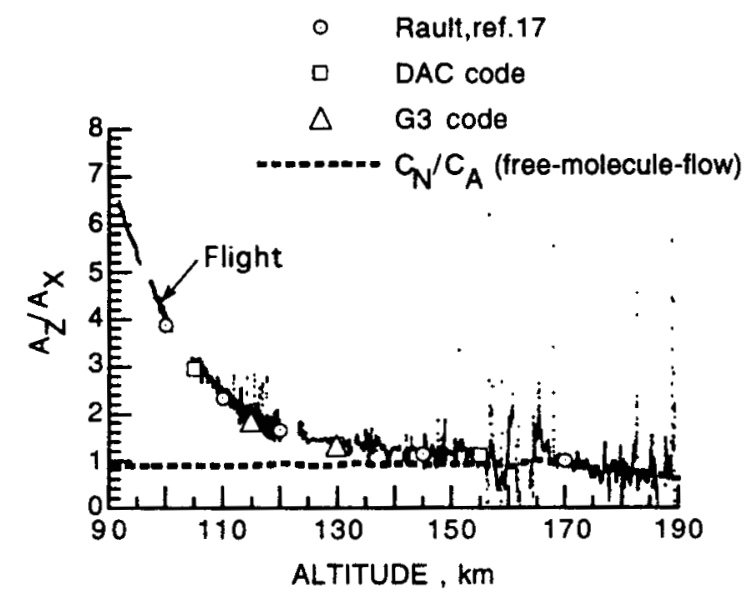

Fig 17 Comparison of flight measured normal to axial coefficient with DSMC simulations. 